Vol. $7, n^{\circ} 1 \mid 2003$

Varia

\title{
Harald Buhlan, Werner Jung (eds.), Wessen Freund und wessen Helfer ? Die Kölner Polizei im Nationalsozialismus
}

Köln, Emos Verlag, 2000, 675 pp., ISBN 3-89705-200-8 (Schriften des Dokumentationszentrums der Stadt Köln, Bd. 7).

\section{Benoit Majerus}

\section{(2) OpenEdition}

\section{Journals}

Édition électronique

URL : https://journals.openedition.org/chs/625

DOI : $10.4000 /$ chs. 625

ISSN : 1663-4837

\section{Éditeur}

Librairie Droz

\section{Édition imprimée}

Date de publication : 1 juillet 2003

Pagination : 143-145

ISBN : 2-600-00865-9

ISSN : $1422-0857$

\section{Référence électronique}

Benoit Majerus, «Harald Buhlan, Werner Jung (eds.), Wessen Freund und wessen Helfer? Die Kölner Polizei im Nationalsozialismus », Crime, Histoire \& Sociétés / Crime, History \& Societies [En ligne], Vol. 7, n¹ | 2003, mis en ligne le 24 février 2009, consulté le 23 mars 2022. URL : http:// journals.openedition.org/chs/625; DOI : https://doi.org/10.4000/chs.625

Ce document a été généré automatiquement le 23 mars 2022

(C) Droz 


\title{
Harald Buhlan, Werner Jung (eds.), Wessen Freund und wessen Helfer? Die Kölner Polizei im Nationalsozialismus
}

\author{
Köln, Emos Verlag, 2000, 675 pp., ISBN 3-89705-200-8 (Schriften des
}

Dokumentationszentrums der Stadt Köln, Bd. 7).

\section{Benoit Majerus}

\section{RÉFÉRENCE}

Harald Buhlan, Werner Jung (eds.), Wessen Freund und wessen Helfer ? Die Kölner Polizei im Nationalsozialismus, Köln, Emos Verlag, 2000, 675 pp., ISBN 3-89705-200-8 (Schriften des Dokumentationszentrums der Stadt Köln, Bd. 7).

Avant même la fin du Troisième Reich il ne faisait plus de doute pour la plus grande partie de la population que ce régime était un état policier. La fameuse Gestapo en devint très vite le symbole. Pendant de longues décennies, les historiens semblaient s'accommoder de cette image d'Épinal. Depuis une quinzaine d'années, l'historiographie a commencé à s'intéresser de plus près à la branche la plus «mythique » de l'appareil de surveillance. Un groupe d'historiens allemands autour de Gerhard Paul et Klaus-Michael Mallmann a proposé une interprétation diamétralement opposée à celle qui dominait jusqu'à ce moment : la Gestapo y est présentée comme une police à personnel réduit et peu puissant, une institution qui réagit face à une société allemande qui s'autocontrôle ${ }^{1}$. En paraphrasant un bon mot de Hans Mommsen sur Hitler (schwacher Führer), on pourrait parler d'une police faible. Ce point de vue ne tarda pas à provoquer des études qui contrebalancent cette thèse ( $c f$. la récente étude de Eric A. Johnson ${ }^{2}$ ). Le débat en cours est le meilleur signe de la vitalité de ce domaine de recherche. Mais on oublie vite que la Gestapo ne représente qu'un aspect de la police opérant pendant le Troisième Reich. 
2 L'ouvrage recensé ici est un des premiers essais à présenter une histoire globale de la police allemande entre 1933 et 1945 et même au-delà, vu que l'après-guerre y est aussi traitée : d'un côté, sous la question des continuités sociologiques et d'un autre côté, sous la question des poursuites judiciaires contre des membres de la police allemande en RFA. Profitant de l'ouverture d'esprit de la police de Cologne, un groupe d'historiens s'est attaché à dépouiller pendant plusieurs années ces archives redécouvertes. Cologne était, au moment où Hitler devint chancelier, la quatrième plus grande ville d'Allemagne.

3 Il serait vain de commenter chacune des vingt-deux contributions; je me limiterai donc aux articles qui me semblent les plus intéressants.

4 Dans un bref, mais instructif survol historiographique, Herbert Reinke (pp. 51-63) énonce les résultats et lacunes de l'actuelle recherche. À la fin de sa contribution, il lance l'hypothèse suivante : la police pendant le Troisième Reich s'est développée en une police $d u$ Troisième Reich. Cette hypothèse pourrait servir de fil rouge à l'étude des différents corps de police sous le régime nazi. Selon Reinke, le travail policier se caractérise encore dans les premières années après 1933 par la continuité d'un système autoritaire; à partir du milieu des années trente, la police allemande participe à un projet racial (p. 61).

5 Non seulement la Gestapo, mais aussi les bataillons de police, ont acquis, depuis les travaux de Christopher R. Browning et Daniel J. Goldhagen ${ }^{3}$, une triste rénommée à cause de leur participation à l'extermination de juifs et de "partisans » sur le front de l'Est. Stefan Klemp et Herbert Reinke ont décidé de s'intéresser à un autre front où ces unités étaient aussi en action, à savoir les Pays-Bas (pp. 263-274). Ils touchent ici à un domaine dans lequel les recherches sont presque inexistantes. Ce déficit s'explique entre autres par l'absence de sources. La justice allemande semble avoir peu enquêté sur ces policiers et les documents judiciaires, qui ont facilité les travaux de Browning, manquent. Les deux auteurs ont cependant réussi à dégager l'implication du bataillon de réserve 68 dans la persécution des juifs néerlandais.

6 À côté de la persécution des juifs, le sort d'autres minorités exclues de la Volksgemeinschaft a récemment attiré l'intérêt des historiens ( $c f$. les travaux de Burkhard Jellonek sur les homosexuels et de Michael Zimmermann sur les tziganes) ${ }^{4}$. Frank Sparing livre une analyse du service de la police criminelle responsable de la persécution des tziganes à Cologne (pp. 519-574). Il souligne d'abord le fait que les tziganes étaient déjà considérés comme dangereux pendant la République de Weimar. Leur caractère criminel ne semble laisser aucun doute, ce qui explique que leur surveillance incombait à la Kriminalpolizei. Ainsi le bureau central pour la lutte contre les tsiganes (Reichszentrale zur Bekämpfung des Zigeunerunwesens) était issu d'un service qui existait depuis la fin des années vingt. Il décrit alors d'une manière détaillée les différentes étapes de l'exclusion de ce groupe de la société allemande qui allait coûter la vie à 25000 tsiganes autrichiens et allemands. Mais ce qui donne plus à réfléchir est la fin de son article : le recensement spécifique des tsiganes après 1945 a été poursuivi sans interruption. Dans les deux Länder de Hambourg et de Hesse, l'enregistrement se basait même partiellement sur les numéros tatoués dans les camps de concentration.

7 Au plus tard depuis la biographie de Ulrich Herbert ${ }^{5}$ sur Best, les historiens se penchent sur les continuités et ruptures des administrations après la guerre. Dans un article bien documenté, Stefan Noethen essaie de répondre à cette question en ce qui concerne la police de Cologne (pp. 575-601). S'il y a des changements à la tête de la police et au 
niveau de l'organisation institutionnelle, les continuités qui existent au niveau du personnel, surtout à partir de la fin des années 40 (où de nombreux anciens policiers sont réintégrés dans la police, faute de personnel qualifié), font pencher la balance plutôt en faveur d'une continuité importante.

8 À côté de ces contributions, de multiples autres aspects sont abordés : analyse de la Machtübernahme en 1933 (Werner Jung, pp. 64-144), survol administratif des différents services de police (Harald Buhlan, pp. 145-197), propagande policière (Willy Hansen, pp. 230-262), implication des bataillons de police dans la persécution des juifs à l'Est (Stephan Klemp, pp. 277-298), fonctionnement de la Kripo à Cologne, poursuite des asociaux et des homosexuels (Thomas Roth, pp. 424-464, Christa Schikovra, pp. 465-491 et Jürgen Müller, pp. 492-518) et les poursuites judiciaires engagées contre des membres de la police après 1945 (Stefan Klemp, pp. 602-618 et Heiner Lichtenstein, pp. 619-632).

9 Le présent volume impressionne par sa taille (675 pages). Cologne sera sûrement encore pour quelques temps la ville où le travail policier pendant les douze années du régime nazi aura été le mieux étudié. L'histoire des différentes institutions qui composent «la" police - aussi bien que celle de son fonctionnement à l'étranger ou celle de leurs victimes - est abordée.

10 L'historiographie sur le sujet s'enrichit de plus en plus de ces monographies locales ${ }^{6}$. Ce volume permettra de reconstruire une nouvelle histoire de la police allemande, "par en bas ». Mais la richesse du volume, c'est-à-dire son exhaustivité, peut aussi poser problème. Même si le livre est issu d'un projet commun, le fil conducteur semble manquer. Les articles se suivent sans qu'une logique (autre que chronologique) relie les différents travaux. Parfois une contribution ressemble à une simple énumération plutôt qu'à une véritable analyse. De plus les différents services de police semblent évoluer en vase clos. Une seule contribution s'intéresse aux relations entre la Gestapo et le reste de la police (Severin Rueseling, pp. 198-229). Finalement on aurait bien aimé voir un intérêt plus prononcé pour les relations que la police entretient avec les autres institutions responsables du maintien de l'ordre (moral, social...) dans une grande ville comme Cologne (p. ex. les services sociaux).

\section{NOTES}

1. Cf. Paul, Gerhard, Mallmann, Klaus-Michael (eds.) Die Gestapo im Zweiten Weltkrieg. «Heimatfront» und besetztes Europa, Darmstadt, 2000, livre dans lequel les deux auteurs essaient de répondre aux critiques qui leur ont été adressées après la publication de leur livre Die Gestapo. Mythos und Realität, Darmstadt, 1995.

2. Eric A. Johhnson, Naziterror: the Gestapo, Jews, and Ordinary Germans, New York, Basic Books, 1999.

3. Christopher R. Browning, Des hommes ordinaires. Le $101^{e}$ bataillon de réserve de la police allemande et la solution finale en Pologne, Paris, Les Belles Lettres, 1994. Daniel J. Goldhagen, Hitler's Willing Executioners. Ordinary Germans and the Holocaust, New York, Knopf, 1996. 
4. Burkhard Jellonek, Homosexuelle unter dem Hakenkreuz. Die Verfolgung von Homosexuellen in Dritten Reich, Paderborn, 1990; Michael, Zimmermann, Rassenutopie und Genozid. Die nationalsozialistische «Lösung der Zigeunerfrage», Hambourg, 1996.

5. Ulrich Herbert, Best: Biografische Studien über Radikalismus, Weltanschauung und Vernunft, 1903-1989, Bonn, 1996.

6. Par exemple Linck, Stefan, Der Ordnung verpflichtet: Deutsche Polizei 1933-1949. Der Fall Flensburg, Paderborn (e.a.), 2000.

\section{AUTEURS}

\section{BENOIT MAJERUS}

benoit.majerus@cegesoma.be 